\title{
Hardware of Monitoring the Identity of Operating Cycles of Diesel Generator
}

\author{
Oleksandr Yenikieiev \\ ORCID 0000-0001-8633-3233 \\ dept. Intelligent Decision Making Systems \\ Donbass State Engineering Academy \\ Kramatorsk, Ukraine \\ yenikieiev@ukr.net \\ Dmitriy Zaharenkov \\ ORCID 0000-0003-3951-022X \\ dept. Intelligent Decision Making Systems \\ Donbass State Engineering Academy \\ Kramatorsk, Ukraine \\ Dmit.zakharen@gmail.com
}

\author{
Liudmyla Nechvoloda \\ ORCID 0000-0002-7584-6735 \\ dept. Intelligent Decision Making Systems \\ Donbass State Engineering Academy \\ Kramatorsk, Ukraine \\ lylyne4v@gmail.com \\ Katerina Krykunenko \\ ORCID 0000-0003-1530-216X \\ dept. Intelligent Decision Making Systems \\ Donbass State Engineering Academy \\ Kramatorsk, Ukraine \\ ladybabenko87@ukr.net
}

\begin{abstract}
It is offered to use a signal of fluctuations of speed of rotation of a cranked shaft at monitoring of identity of working cycles of the power unit. Hardware has been built and information technology for primary signal processing has been created. A deterministic mathematical model of a diesel circuit in the form of a mechanical system with four degrees of freedom under the condition of friction, Laplace transform, methods of similarity theory and signal graph theory, as well as the method of determinants is used in the development of algorithmic software. The monitoring procedure leads to the solution of a system of redefined algebraic equations with optimization of results based on the method of least squares.
\end{abstract}

Keywords-fluctuation signal, information technology, algorithmic software, Laplace Transform

\section{INTRODUCTION}

Technical and economic indicators of the diesel generator (DG) determine the setting of operating cycles [5], which depends on the uncertainty of setting the phases of gas distribution $[6,7]$. The use of hardware for software setting of these phases allows to reduce the uncertainty of setting the identity of work cycles. Solving this problem will provide fuel savings of 5\% [2] and reduce the likelihood of overloading individual cylinders. Therefore, the choice of the method of measuring the input information, construction of hardware and algorithmic monitoring of the identity of the operating cycles is an urgent scientific and applied task.

\section{ANALYSIS OF LITERARY DATA}

The authors propose the idea of monitoring the identity of the operating cycles of DG 3TD-1 based on the processing of data of indirect measurements. As a signal of measuring information, the authors chose fluctuations in the speed of rotation of the crankshaft. The results of studies of the signal of non-uniformity of rotation of the crankshaft DG 6NVD48UA are given in [4]. A measuring transducer and a method for calculating the average effective cylinder pressure, engine power, excess air ratio and exhaust gas temperature have been developed. In [3], a method was proposed to improve the control uncertainty of the fuel supply process to the cylinders based on measurements of the amplitude of angular velocity oscillations and phase shifts of their extremes relative to the top dead center of the first cylinder. Reduces the effect of random interference on the information signal of the non-uniformity of the crankshaft rotation of the high-pass filter with a finite impulse response [1]. In this research the technique of processing of a signal of fluctuations with use of possibilities of the Matlab software environment is developed.

Known hardware and software tools for monitoring the identity of operating cycles have unsatisfactory metrological characteristics and low productivity of input information processing, as well as there is no algorithmic and applied software for processing indirect measurement data. The aim of research is to reduce the uncertainty of the hardware for measuring the signal of the crankshaft speed fluctuations and to improve the productivity of the process of estimating the identity of the working cycles of the DG.

\section{CONSTRUCTION OF HARDWARE}

The authors used electro-hydraulic injectors as actuators for adjusting the processes of fuel and air supply to the cylinders. Synchronization of the hardware with the phase of rotation of the crankshaft provides a signal of the top dead center of the first cylinder. The block diagram of the hardware for monitoring the identity of the operating cycles of the power unit based on the processing of data of indirect measurements is presented at Fig. 1. The architecture of the system consists of the following components: instantaneous crankshaft speed sensor (SS), top dead center sensor of the first cylinder (TDCS), measuring transducer (MT) of the fluctuation signal, microcomputer and three actuators (ACT1, ACT2 and ACT3). 


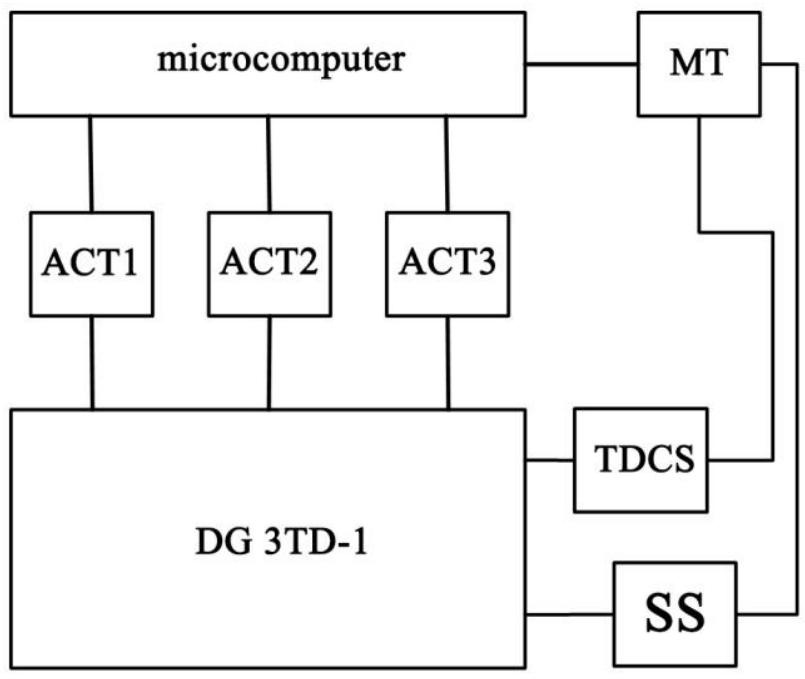

Fig. 1. Hardware architecture

The procedure for monitoring the identity of the operating cycles of DG 3TD-1 involves the following actions:

- measurement of the crankshaft speed signal;

- averaging of the received information and formation of a sample within one turn of a cranked shaft;

- obtaining an array of discrete values of the fluctuation signal;

- construction of a mathematical model of the steep circuit DG 3TD-1;

- solving a deterministic system of differential equations of mass motions in order to monitor the identity of operating cycles;

- establishment of deviations of the working process of the power unit;

- formation of ACT1, ACT2 and ACT3 software control signals.

A method for measuring the signal of fluctuations of the crankshaft speed DG is proposed, which due to the use of hardware to compensate for the kinematic uncertainty of the primary transducer provides the required accuracy. The method consists in the construction of hardware for processing frequency-modulated signal based on multichannel measurements of time intervals, which are formed by the selected line of the primary converter and correspond to the full rotation of its shaft. In this case, its kinematic uncertainty of the primary converter does not affect the duration of the periods of the output frequency-modulated signals. Based on the processing of experimental data using the information approach, it was found that multi-channel measurements of crankshaft rotation periods significantly reduce the uncertainty of fluctuation signals. Based on this method, MT was developed.

\section{TORQUE CIRCUIT SIMULATION}

Subject to friction and oscillations between the masses, the torsional scheme of the power unit is presented in the form of a mechanical system with four degrees of freedom.
The dynamics of rotation of the cylindrical masses of the crankshaft is described by a system of linear differential equations of this kind:

$$
J_{i} \varphi_{i}^{\prime \prime}+\beta \varphi_{i}^{\prime}-\frac{\varphi_{i+1}-\varphi_{i}}{e}+\frac{\varphi_{i}-\varphi_{i-1}}{e}=M_{i}
$$

where $i=1,2, \ldots 4 ; e$ is pliability of relations between the masses; $M_{i}(t)$ is torque obtained as a result of processing of indicator diagrams and compression diagrams, without taking into account the average value; $\varphi_{i}(t)$ is angle of rotation of the mass; $J_{i}$ is moment of inertia of the mass; $\beta$ is friction.

The system of differential equations (1) is reduced to a dimensionless form by means of similarity theory theorems. The Laplace transform under zero initial conditions gives this system the following form:

$$
\left(J_{i} e p^{2}+\beta e p+2\right) \varphi_{i}-\varphi_{i+1}-\varphi_{i-1}=e M_{i}
$$

After mathematical transformations, the system of equations (2) takes the following form:

$$
\varphi_{1}=\sum_{j=1}^{3} \frac{\Delta_{j}}{\Delta} M_{j}=\sum_{j=1}^{3} W_{j} M_{j}
$$

where $\varphi_{1}(p)$ - is the Laplace transform of the speed signal of the first mass; $M_{j}(p)$-torque; $\Delta, \Delta_{j}$ - determinants of the system of equations; $W_{j}-$ transmission functions that establish information links between the torques of individual cylinders and the signal of fluctuations in the speed of rotation of the first mass. Determinants were obtained using the Mathcad software environment.

The calculation of the CFC of the crankshaft-cylinder paths was performed using the capabilities of the Matlab software environment. As a result of the analysis of LFA, it was concluded that they have an intersection point and in the frequency band $9-50 \mathrm{~Hz}$ there are oscillations. The roots of numerators and denominators of transfer functions are obtained. The establishment of information links between the torques of the cylinders and the signal of fluctuations of the speed of rotation of the first mass is also carried out on the basis of the use of the topographic rule of the theory of signal graphs. Special points of transfer functions are established.

Algorithmic support is based on solving a system of algebraic equations:

$$
B D=\varphi_{1}-\varphi_{1,0}
$$

where B - the matrix, the coefficients of which are determined on the basis of the transfer functions and torques of the cylinders, depending on the chosen calculation method; D - vector-column of coefficients; $\varphi_{1}$ - vector- 
column of the signal of measurement information; $\varphi_{1,0}$ vector column of the fluctuation signal of the first mass of the crankshaft in the absence of fuel supply to the cylinders.

This signal is calculated in advance on the basis of processing of compression diagrams of cylinders for the purpose of receiving frequency representation of torques and the received transfer functions. Its time representation can be obtained in the form of the sum of the convolution integrals between the torque of the cylinder and the weight function, which is set on the basis of the transmission.

The number of equations in system (4) is determined by the number of harmonic components of the frequency representation of torque. Accordingly, system (4) is overridden, so the software uses the least squares method to optimize the solution. The number of sampling intervals of the signal of fluctuations of the rotational speed of the first mass is chosen using Kotelnikov's theorem. Computer simulations of the effects of random interference on the uncertainty of the calculation of coefficients have been performed $D_{i}$. The influence of additive interferences of hardware on the signal of measuring information, additive technological interferences of phase delays of cylinders concerning the first, and also at known additive interference of a signal of fluctuations for change of width of a spectrum of its frequency representation is investigated. Appropriate graphs are constructed, with the help of which the requirements to the uncertainty of the measurement information signal are formulated.

In the temporal representation of the measurement information, the system of algebraic equations (4) is the balance between the values of the fluctuation signal of the first mass at specific points in time and the corresponding sum of the calculated contributions of each cylinder. The addition to the amount is determined by the product of the coefficients $D_{i}$ on the corresponding weight function of the contribution of the cylinder to the fluctuation signal. This function is calculated in advance as the convolution interval between the torque of the individual cylinder and the corresponding transfer function. For this purpose, the algorithmic support for monitoring the operating cycles of DG implements the appropriate computational procedures.

Algorithmic support for monitoring the identity of the operating cycles of DG 3TD-1 is based on the use of time and frequency representation of the signal of measuring information. It is established that their use provides a fairly close performance of calculations. If random information is measured on the signal of the measurement information, preference should be given to the use of the algorithm for obtaining estimates of the maximum likelihood of the coefficients of the cylinders.

\section{CONSOLUTION}

The concept of software movements of hardware for controlling the processes of fuel and air supply to the cylinders DG, a feature of which is the use of indirect measurement data to monitor the identity of operating cycles.
The hardware uses a signal of measuring information in the form of fluctuations in the speed of rotation of the crankshaft, and also has a device for synchronization with the operating cycle of the first cylinder.

The representation of the torsional scheme of DG in the form of a deterministic mechanical system with four degrees of freedom is offered. The dynamics of mass motions of a mathematical model taking into account friction is described by a system of second-order differential equations, the parameters of which are normalized on the basis of similarity theory theorems. The mathematical apparatus of the Laplace transform was used to solve it

The transfer functions which establish information communications between torques of cylinders and a signal of fluctuations of speed of rotation of the first weight of a cranked shaft are received. It is established that the use of determinants and methods of signal graph theory give almost identical expressions for transfer functions. The scheme of computer modeling of the signal of measuring information is constructed and on the basis of using the method of the model which is studied, the procedure of identification of its parameters is carried out.

Computer modeling of the computational procedure for establishing the coefficients of cylinders under the action of random algebraic equations on the system is carried out. Algorithmic and application software for computer simulation of noise has been developed. Graphs of uncertainties of calculation of identity of working cycles of DG are received. Requirements for the uncertainty of the VP unit have been established.

\section{REFERENCES}

[1] Bodnar B.E., Okskashov O.B., Chernyayev D.V. Determination of the method of filtration of the signal of uneven frequency of the rotation of the crankshaft of the diesel engine. Visnyk DNUZT [Bulletin of DNURT], 2013, i 1(43), pp. 113-118. (in Ukrainian)

[2] Borisenko Ye.M., Yenikieiev O.F. Information technology for estimating the identity of the working cycles of internal combustion engines. Informatsiyni tekhnolohiyi ta kompyuterna inzheneriya [Information technology and computer engineering], 2016, no 2, pp. 21-28. (in Ukrainian)

[3] Grachev V.V. Experimental estimation of diagnostic method of diesel engines due to uneven rotation of the crankshaft. Progressivnie processi technologicheskoy ekspluatacii avtomiobiley [Progressive processes of technological operation of cars], Moskva, 1982, pp. 4650. (in Russian)

[4] Pokusayev M.N., Sibiryakov K.O., Shevchenko A.V. Experimental determination of the degree of irregularity of the collapse of the shaft of the machine-propulsion complex of the ship 1557. Vestnik AGTU [Bulletin of AGTU], 2008, no 2(43), pp. 140-144. (in Russian)

[5] Challen B., Baranescu R. Diesel Engine Reference Book. Butterworth-Heinemann, 1999. 682 p.

[6] Gawande S.H., Navale L.G., Nandgaonkar M.R., Butala D.S., Kunamalla S. Cylinder Imbalance Detection of Six Cylinder DI Diesel Engine Using Pressure Variation. International Journal of Engineering Science and Technology, 2010, vol. 2(3), pp. 433-441.

[7] Gawande S.H., Navale L.G., Nandgaonkar M.R., Butala D.S. Harmonic Frequency Analysis of Multi-Cylinder Inline Diesel Engine Genset for Detecting Imbalance. International Review of Mechanical Engineering, 2009, vol. 3 no 6, pp. 782-787. 